\title{
The Use of Moringa oleifera Seeds as Absorbent in Reducing Groundwater Hardness.
}

\author{
E. Fosso-Kankeu, T. Motsosi, S. Pandey, F. Waanders, and A.C. Eloka-Eboka
}

\begin{abstract}
Leaching due to landfills has proven to be one of the major sources of pollutants in groundwater. Waste materials get dissolved and liquified to form leachates, with constituent organic, inorganic, and heavy metals inherent. There are no working alternatives for discarding wastes without direct or indirect contamination of the groundwater. There is therefore the need to consider green, economic, viable, and sustainable purification techniques to be adopted in polluted groundwater systems.

Recent studies have shown that Moringa oleifera seeds possess huge potentials as absorbent and edible in nature and can be used as both a coagulant and flocculant in the water purification suited for developing countries like South Africa. This study thus draws inference at investigating the potential Moringa oleifera seeds have on reducing groundwater hardness.

The metals of interest for reduction are calcium and magnesium ions. This was accomplished by particle size characterization of the prepared ground seeds which was subjected to FTIR characterization and thereafter absorption studies were conducted on collected groundwater with known metal composition. Results obtained indicated that the total percentage of absorbed calcium and magnesium after 240 minutes were $55 \%$ and $31 \%$, respectively. There are three binding groups in the matrixes of Moringa oleifera seed structure as determined by the absorption studies. The overall implication is that the seeds of Moringa have the potential to reduce groundwater hardness.
\end{abstract}

Keywords-absorbent, calcium, Groundwater, Landfills, magnesium, Moringa oleifera

\section{INTRODUCTION}

Life sustainability depends directly on the stable supply of drinkable water in addition to other key indicators. There are two reliable sources of water, which are groundwater and surface water. Both water sources need some form or series of

Manuscript received October 01, 2020. This work was supported in part by the North- West University, Potchefstroom campus faculty of engineering (chemical engineering department)

E. Fosso-Kankeu is with the Water Pollution Monitoring and Remediation Initiatives Research Group, School of Chemical and Minerals Engineering, North-West University, Potchefstroom, South Africa.

S. Pandey is with the Water Pollution Monitoring and Remediation Initiatives Research Group, School of Chemical and Minerals Engineering, North-West University, Potchefstroom, South Africa.

F. Waanders is with the Water Pollution Monitoring and Remediation Initiatives Research Group, School of Chemical and Minerals Engineering, North-West University, Potchefstroom, South Africa.

A.C. Eloka-Eboka is with the Water Pollution Monitoring and Remediation Initiatives Research Group, School of Chemical and Minerals Engineering, North-West University, Potchefstroom, South Africa. purification before consumption depending on the initial condition(s). Pollutants are found in water bodies due to several anthropogenic activities including domestic, industrial, and natural activities [1-4]. This study focuses on groundwater and its purification method(s). Groundwater supplies approximately 2 billion human inhabitants/populations with water daily [1] over the world.

Groundwater is an underground water which flows through pores and cracks in soil and rocks to form an aquifer. They are also formed from run-offs from rains and other forms of precipitation. It provides drinking water, agricultural irrigation, and industrial process water. It also feeds rivers, lakes, and wetlands. The main source of groundwater is rain, which means there is a differential amount of groundwater occurring during the dry and wet seasons. Groundwater provides about half of the drinking water in the world.

The main groundwater pollutants are nitrates $\left(\mathrm{NO}_{3}{ }^{-}\right)$. Other pollutants are heavy metals (e.g. $\mathrm{Fe}, \mathrm{Cu}, \mathrm{Cr}, \mathrm{Pb}$, and $\mathrm{Zn}$ ). Sources of nitrates in groundwater are agricultural activities emanating from fertilizers, manures and legumes, atmospheric sources, and septic systems. Most rural parts of South Africa are facing a shortage of water due to spells and climatic locations and therefore constitute one of the cardinal challenges of livelihoods. Minimal attention has been given to the contribution of groundwater on water security [6] in Africa.

Leaching due to landfills has also proven to be one of the major sources of pollutants in groundwater. Waste materials form the liquids which are called leachates, and they constitute organic, inorganic, and heavy metals [7] inherent. Some factors affect the characteristics of leachates. The age of the landfill and the hydrology of the landfill are the main factors [8]. The age of the landfill might affect the leachate in such a manner that the higher the age, the more there is the accumulation of wastes. Hydrology of the landfill might be described as how far groundwater is with reference to the wastes leaching in. Most developing countries like South Africa rely on waste disposal as a means of handling landfills. There is no working alternative for dumping wastes without polluting the groundwater. The need to develop, economic, sustainable, and viable purification techniques cannot be under-estimated and therefore such an approach must be employed as much as possible.

Moringa oleifera is a tree that is from a family Moringaceae [9]. It is an indigenous plant of Africa, India, Asia, Pakistan, and Arabia [10]. Furthermore, the Moringa tree has other local names like Benozolive, Horseradish tree, Marango, and Mlonge 
across some of these countries of origin. Moringa trees can withstand drought even at hot conditions, they do not need soil rich with nutrients for survival. They can thrive on any marginal land whatsoever. They are planted around the world and are utilized extensively in medicine, food, and other industrial applications. The oils from the seeds are rich in essential fatty acids including oleic, linoleic, palmitic, and other carboxylic acids.

Recent studies have shown that Moringa oleifera seeds are not poisonous, they are edible and can be used as coagulants and flocculants in water purification especially in developing countries like South Africa [11]. That is why Moringa oleifera could be considered as an alternative green solution since it is cheap and available in the five provinces in South Africa including Limpopo, Free State, Mpumalanga, KwaZulu-Natal, and Gauteng.

Furthermore, this study is geared towards harnessing the green potential of Moringa in water purification and thus contributing in reducing or eliminating the shortage of portable water especially in remote areas [12] while at the same time, deploying its capability as an absorbent to reduce hardness in water/underground water by coagulating metal ions causing hardness. The goal is to ease the process of purification of water by rural people or people residing in remote urban areas so that access to portable and drinkable-water will be all-encompassing. Most importantly, to be able to meet the World Health Organization standards in providing affordable portable water, the study is timely.

Overall, the employability of Moringa oleifera seeds as a green sustainable material for water purification is motivated by the fact that it is an affordable natural absorbent capable of reducing/eliminating water hardness and other harmful pollutants. Moringa as an absorbent is locally available in South Africa and elsewhere in Africa. The technology to be adopted in the utilization of Moringa for water treatment is easy and accessible to deploy; both for domestic and commercial scale to the extent of individual home use on a small scale. To the best of the authors' knowledge, Moringa oleifera seeds have never been applied for the treatment of landfill ground water.

\section{MATERIALS AND METHODS}

\section{A. Sample Collection and Preparation}

Moringa oleifera seeds were collected from a village in Limpopo province and experimentally prepared. Groundwater for absorption assessment was collected from Potchefstroom landfill, North West province of South Africa.

Collected groundwater was stored in the refrigerator so that no microbial growth occurs. The seeds were crushed using a jaw crusher, followed by particle size characterization. They were sieved using a 150 microns screen. The underflow was retrieved for experiment purposes. Crushed seeds were put into an oven at a temperature of 70-degree Celsius for 48 hours. This was done so that the seeds are stored dry so that no mold growing.

\section{B. Fourier-Transform Infrared Spectroscopy Characterization}

Fourier transform infrared (FTIR) spectroscopic analysis was conducted to investigate the binding groups present in the seeds of Moringa. To perform FTIR analysis, $20 \mathrm{mg}$ of sample is required with $200 \mathrm{mg}$ of potassium bromide. Potassium bromide and the prepared seeds were weighed and mixed until a homogenous mixture is obtained. The mixture is put into the analyzer such that the infrared radiation shines directly onto the sample. Then results in form of a graph are displayed showing molecular vibrations as a result of the absorption of the infrared radiation. The model of the equipment used is IR Affinity-1 FTIR-S/N A21374902809CZ (US).

\section{Absorption Experiment}

Four dosages $(0.05,0.1,0.15$, and 0.2 grams) of powdered seeds were used so that the most effective (optimum) one can be determined. The Moringa absorbents were dosed into $50 \mathrm{~mL}$ of groundwater. Firstly, a thermometer was used to make sure that the temperature of the water was 25 degrees Celsius. The adsorbent samples and water mixture were kept in Erlenmeyer flasks. The flasks were put into the orbital shaking incubator model labcon 5082U at 25 degrees Celsius and $160 \mathrm{rpm}$ for 90 minutes. The experiments were done in duplicates.

After 90 minutes, the samples were transferred into the centrifuge tube and inserted into the centrifuge model Hettich Zentrifugen Rotofix 32 A used to settle the adsorbent particles at the bottom of the tube.

For spectroscopic analysis, the supernatant was collected by pipette and put into another tube, subjected to Inductively Coupled Plasma (ICP) analysis (Agilent Technologies, USA). On obtaining the results, the dosage that showed better purification capacity was chosen as the optimum dosage. In this experiment, $0.2 \mathrm{~g}$ was the optimum dosage.

Experiments were repeated using $2 \mathrm{~g}$ as dosage into $50 \mathrm{ml}$ of groundwater. The effect of time was studied. The following times were the period used :10, 30, 60, 90,120,150,180, 210 and 240 minutes respectively. Results were tabulated and analysed.

\section{RESULTS AND DISCUSSION}

The absorption of metal calcium and magnesium shows two distinct behaviours. Calcium absorption increases with time whereas magnesium absorption decreases with time. This implies that magnesium absorbs very quickly, and desorption occurs afterward.

The total percentage of absorbed calcium after 240 minutes is $55 \%$ and $31 \%$, respectively.

The following equations were used to compute absorption capacity at equilibrium time and absorption capacity at time t, respectively $[13,14]$.

$$
q_{\theta}=\frac{\left(C_{0}-C_{\theta}\right) V}{m}
$$

$\mathrm{q}_{\mathrm{e}}$ - equilibrium absorption capacity

$\mathrm{C}_{\mathrm{o}}$ - initial concentration of metal.

$\mathrm{C}_{\mathrm{e}}$ - equilibrium concentration of metal. 
V- Volume of solution.

$\mathrm{m}$ - Mass of absorbent in solution.

$$
q_{t}=\frac{\left(C_{0}-C_{t}\right) V}{m}
$$

$\mathrm{q}_{\mathrm{t}}$ - absorption capacity at time $\mathrm{t}$.

$\mathrm{C}_{\mathrm{o}}$ - initial concentration of metal.

$\mathrm{C}_{\mathrm{t}}$ - concentration of metal at time $\mathrm{t}$.

$\mathrm{V}$ - Volume of solution.

$\mathrm{m}$ - Mass of absorbent in solution

Pseudo-first and pseudo-second-order kinetic models were used for kinetics studies. The following are relevant linearized equations.

$$
\ln \left(q_{e}-q_{\mathrm{t}}\right)=\ln q_{e}-k t
$$

$\mathrm{q}_{\mathrm{e}}$ - equilibrium absorption capacity.

$\mathrm{q}_{\mathrm{t}}$ - absorption capacity at time $\mathrm{t}$.

k- Constant.

t- Time.

$$
\frac{t}{q_{t}}=\frac{1}{k q_{g}^{2}}+\frac{1}{q_{g}} t
$$

$\mathrm{q}_{\mathrm{t}}$ - absorption capacity at time $\mathrm{t}$.

$\mathrm{q}_{\mathrm{e}}$ - equilibrium absorption capacity.

k- Constant.

t- Time.

Figures 1 and 2 show the experimental data and the one fitted using the pseudo-first and pseudo-second-order for calcium absorption. The data fits better to the pseudo-second-order with an coefficient of determination $\left(\mathrm{R}^{2}\right)$ value of 0.88 , compared to the pseudo-first-order which has a coefficient of determination value of 0.67 . It could be therefore concluded that a chemisorption mechanism is predominant in the uptake of calcium by Moringa olifeira seeds [13, 14]. The equilibrium absorption capacity according to the pseudo-second-order is $20.6 \mathrm{mg} / \mathrm{g}$.

Figures 3 and 4 show the experimental data and the one fitted using the Pseudo-first and pseudo-second-order for magnesium absorption. The data fits best with pseudo-first-order with coefficient of determination value of 0.99 , compared to a value of 0.97 for the pseudo-first order. The equilibrium absorption capacity for magnesium absorption is $29.1 \mathrm{mg} / \mathrm{g}$ according to the pseudo-first-order data fit. While the equilibrium absorption capacity according to the pseudo-second-order is $13.1 \mathrm{mg} / \mathrm{g}$.

The values of the equilibrium absorption are acceptable looking at the concentration value of the absorbent $(0.004 \mathrm{~g} / \mathrm{ml})$.

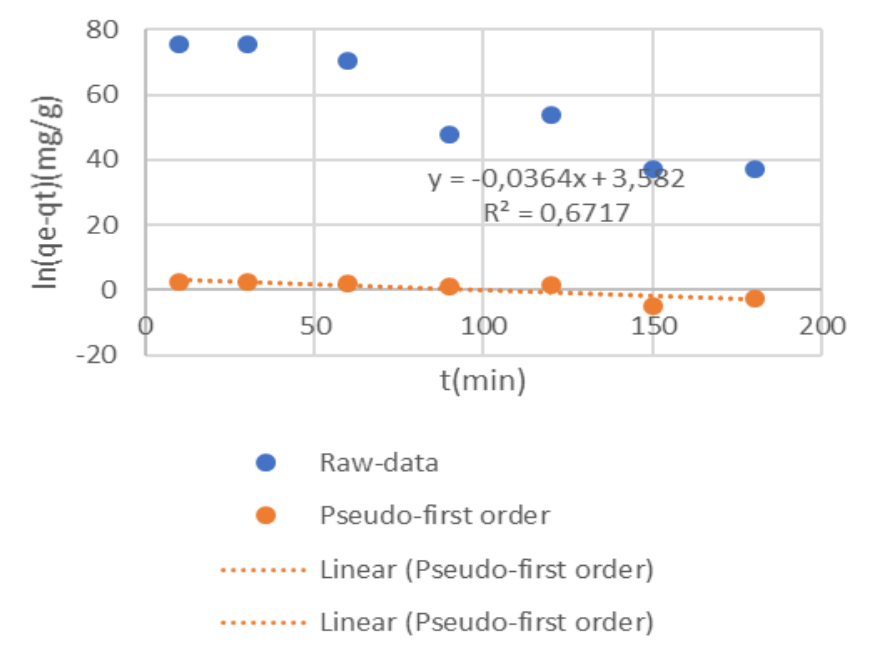

Fig. 1: Experimental data of calcium absorption fitted with pseudo-first-order kinetics formula.

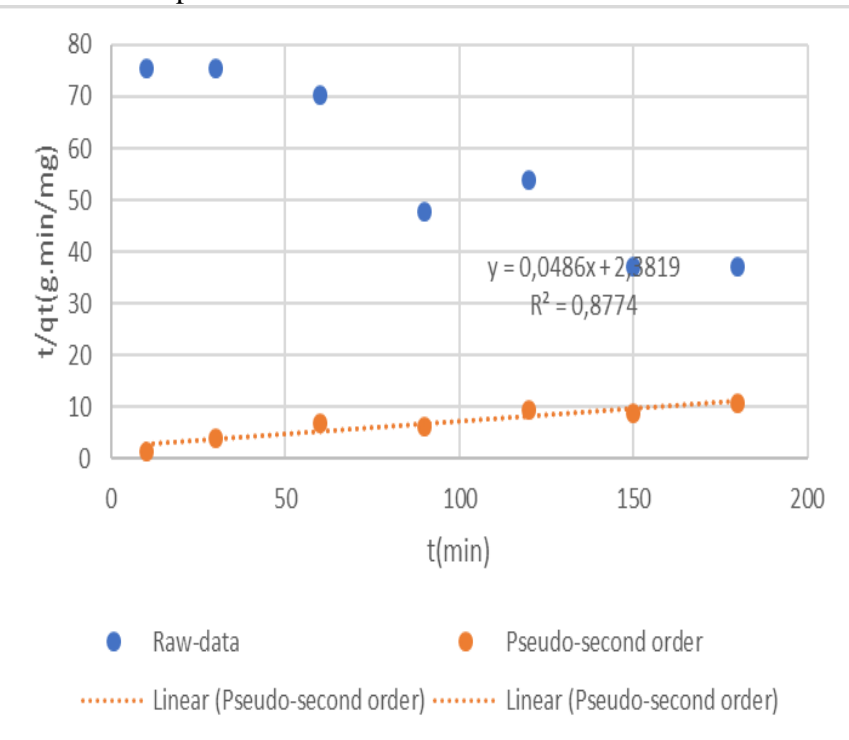

Fig. 2: Experimental data of calcium absorption fitted with a pseudo-second-order kinetics formula.

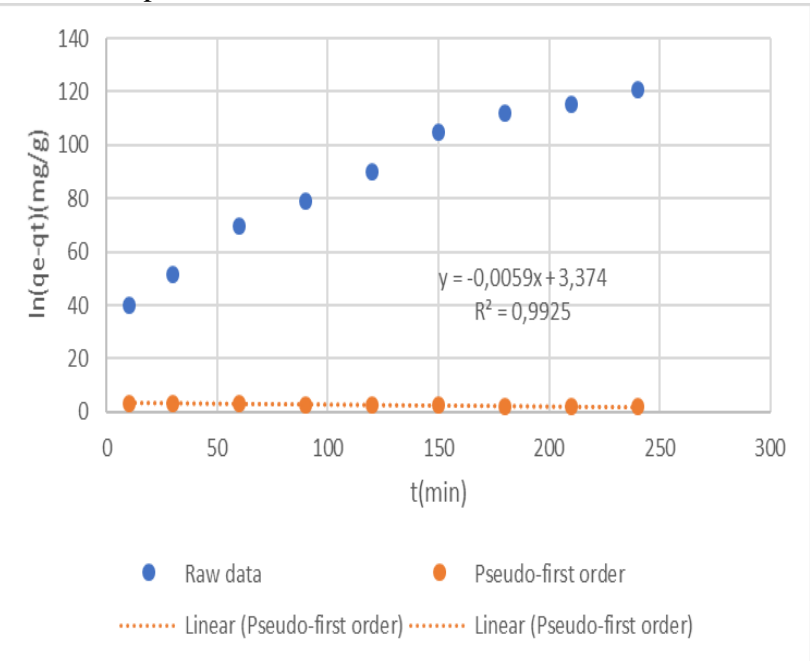

Fig. 3: Experimental data of magnesium absorption fitted with pseudo-first-order kinetics formula. 


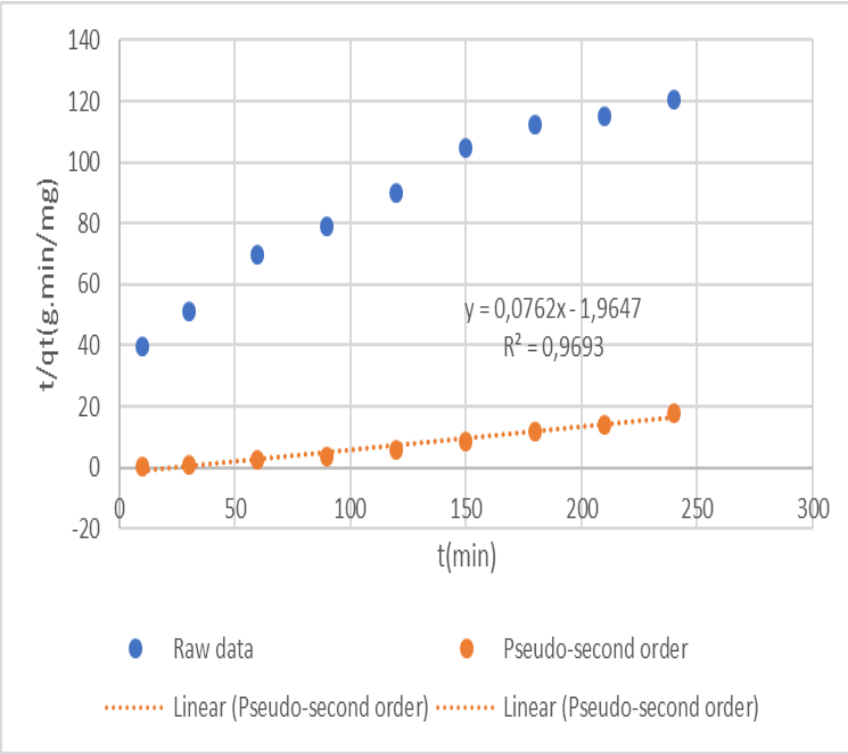

Fig. 4: Experimental data of magnesium absorption fitted with pseudo-second-order kinetic formula.

Looking at Figure 5 the following can be deduced.

In zone 1 between 3700 and $3200 \mathrm{~cm}^{-1} \mathrm{O}-\mathrm{H}$ bond is observed stretching from 3675 to $3425 \mathrm{~cm}^{-1}$. The broad intensity varying between 1250 and 800 is observed. Therefore, Moringa oleifera seeds have an alcohol binding group.

Zone $2\left(3200-2700 \mathrm{~cm}^{-1}\right)$ illustrates the alkane spectrum; the binding group is observed in the range of 3000 to $2840 \mathrm{~cm}^{-1}$. The varying intensity between 500 and 780 occurs. Therefore, the alkane group is present as a binding group in the Moringa oleifera seeds.

Zone $3\left(2300-2100 \mathrm{~cm}^{-1}\right)$ shows the range whereby the intensity variation of the alkyne group should be observed. There are no significant bends in the intensity graph. Therefore, the alkyne group is not present as a binding group in the Moringa oleifera seeds.

Zone $4\left(1950-1650 \mathrm{~cm}^{-1}\right)$ shows the spectrum of the aldehyde functional group. The bend is seen between 1775 and 1720 $\mathrm{cm}^{-1}$. The intensity range of 620 to 480 , showing that the aldehyde group is present as a binding group in the Moringa oleifera seeds.

Zone 5(1680-1450) shows the zone that contains the alkane functional group intensity variation. There is no significant change in the intensity graph. Since there are no significant bends it can be concluded that there is no alkane binding group in the Moringa oleifera seeds.

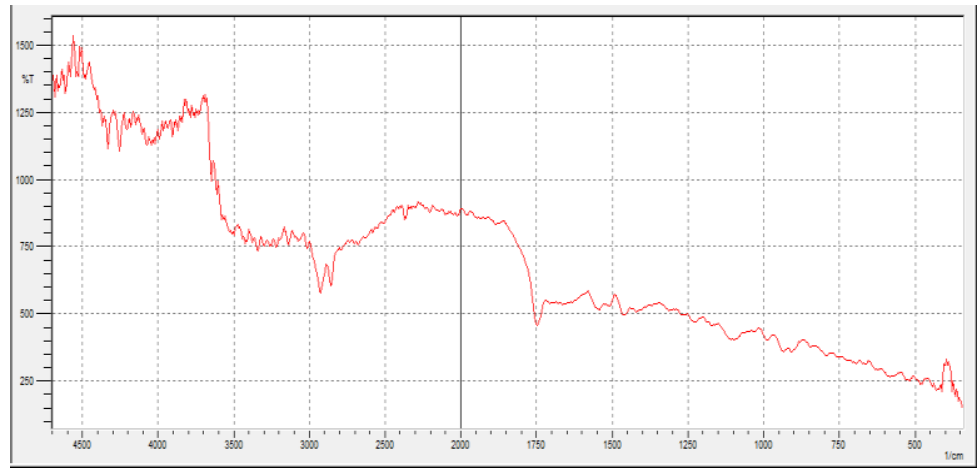

Fig. 5: Fourier-transform infrared spectroscopy for Moringa seeds.

\section{CONCLUSION}

It can be concluded that the Moringa oleifera seeds have the potential to be used as a locally available absorbent to reduce groundwater hardness by looking at the metal removal efficiency. There are three binding groups in the matrixes of Moringa oleifera seed structure as determined by the absorption studies. The overall implication is that the seeds of Moringa has potential to reduce groundwater hardness.

\section{ACKNOWLEDGMENT}

Author EFK acknowledged the financial support from the National Research Foundation (NRF) in South Africa (Grant No: 120323); Any opinion, findings and conclusions or recommendations expressed in this material are those of the authors and therefore the NRF does not accept any liability in regard thereto.

\section{REFERENCES}

[1]E. Fosso-Kankeu, AF Mulaba-Bafubiandi, BB Mamba and TG Barnard. 2011. Prediction of metal-adsorption behaviour in the remediation of water contamination using indigenous microorganisms. Journal of Environmental Management, 92 (10), pp 2786-2793 https://doi.org/10.1016/j.jenvman.2011.06.025

[2]E. Fosso-Kankeu, H. Mittal, F. Waanders, I.O. Ntwampe, S.S. Ray. 2016. Preparation and characterization of gum karaya hydrogel nanocomposite flocculant for metal ions removal from mine effluents. International Journal of Environmental Science and Technology. 13: 711-724. https://doi.org/10.1007/s13762-015-0915-x

[3] E. Fosso-Kankeu, F. Waanders, C.L. Fourie. 2016. Adsorption of Congo Red by surfactant-impregnated bentonite clay. Desalination and Water Treatment. doi: 10.1080/19443994.2016.1177599: 1-9. https://doi.org/10.1080/19443994.2016.1177599

[4] Elvis Fosso-Kankeu, Frans B. Waanders, Frederik W. Steyn. 2017. Removal of $\mathrm{Cr}(\mathrm{VI})$ and $\mathrm{Zn}$ (II) from an aqueous solution using an organic-inorganic composite of bentonite-biochar-hematite. Desalination and Water Treatment. 59: 144-153. https://doi.org/10.5004/dwt.2017.0059

[5] Negi, P., Mor, S., and Ravindra, K., 2018. Impact of landfill leachate on the groundwater quality in three cities of North India and health risk assessment. Environment, Development, and Sustainability, 22(2), pp.1455-1474. https://doi.org/10.1007/s10668-018-0257-1

[6] Calow, R., MacDonald, A., Nicol, A., and Robins, N., 2010. Ground Water Security and Drought in Africa: Linking Availability, Access, and Demand. Ground Water, 48(2), pp.246- 256. https://doi.org/10.1111/j.1745-6584.2009.00558.x

[7] Javahershenas, M., Nabizadeh, R., Alimohammadi, M., and Mahvi, A., 2020. The effects of Lahijan landfill leachate on the quality of surface and 
groundwater resources. International Journal of Environmental Analytical Chemistry, pp.1-17.

https://doi.org/10.1080/03067319.2020.1724984

[8] Jhamnani, B., and Singh, S., 2009. Groundwater Contamination Due To Bhalaswa Landfill Site.

[9] Muyibi, S., and Evison, L., 1995. Moringa oleifera seeds for softening hardwater. Water Research, 29(4), pp.1099-1104. https://doi.org/10.1016/0043-1354(94)00250-B

[10] Anwar, F., Latif, S., Ashraf, M., and Gilani, A., 2006. Moringa oleifera: a food plant with multiple medicinal uses. Phytotherapy Research, 21(1), pp.17-25. https://doi.org/10.1002/ptr.2023

[11] Amagloh, F., and Benang, A., 2009. [online] Sswm.info. Available at: https://sswm.info/sites/default/files/reference_attachments

[12] Brown, J., and Sobsey, M. (2009). Microbiological effectiveness of locally produced ceramic filters for drinking water treatment in Cambodia. Journal of Water and Health, 8(1), pp.1-10. https://doi.org/10.2166/wh.2009.007

[13] Elvis Fosso-Kankeu, Hemant Mittal, Frans Waanders, Suprakas Sinha Ray. 2017. Thermodynamic properties and adsorption behaviour of hydrogel nanocomposites for cadmium removal from mine effluents. Journal of Industrial and Engineering Chemistry. 48: 151-161. https://doi.org/10.1016/j.jiec.2016.12.033

[14] A Leudjo Taka, E Fosso-Kankeu, K Pillay, X Yangkou Mbianda. 2018. Removal of cobalt and lead ions from wastewater samples using an insoluble nanosponge biopolymer composite: Adsorption isotherms, kinetics, thermodynamics and regeneration studies. Environmental Science and Pollution Research. 25(22): 21752-21767.

https://doi.org/10.1007/s11356-018-2055-6 